Revue

d'ethnoécologie
Revue d'ethnoécologie

$11 \mid 2017$

Varia + dossier "Cartographie participative" (2)

\title{
Des savoirs locaux aux experts naturalistes
}

La reconnaissance des savoirs de pêcheurs artisanaux de Martinique

From local knowledge to naturalists experts: The recognition of artisanal fishermen knowledge in Martinique

\section{Myriam Thirot, Pierre Failler et Justin Daniel}

\section{OpenEdition}

\section{Journals}

Édition électronique

URL : http://journals.openedition.org/ethnoecologie/2916

DOI : 10.4000/ethnoecologie.2916

ISSN : 2267-2419

Éditeur

Laboratoire Eco-anthropologie et Ethnobiologie

\section{Référence électronique}

Myriam Thirot, Pierre Failler et Justin Daniel, « Des savoirs locaux aux experts naturalistes », Revue d'ethnoécologie [En ligne], 11 | 2017, mis en ligne le 03 juillet 2017, consulté le 20 avril 2019. URL : http://journals.openedition.org/ethnoecologie/2916; DOI : 10.4000/ethnoecologie.2916

Ce document a été généré automatiquement le 20 avril 2019

\section{(†) $\ominus$}

Revue d'ethnoécologie est mis à disposition selon les termes de la licence Creative Commons Attribution - Pas d'Utilisation Commerciale - Pas de Modification 4.0 International. 


\title{
Des savoirs locaux aux experts naturalistes
}

\author{
La reconnaissance des savoirs de pêcheurs artisanaux de Martinique ${ }^{1}$ \\ From local knowledge to naturalists experts: The recognition of artisanal \\ fishermen knowledge in Martinique
}

Myriam Thirot, Pierre Failler et Justin Daniel

\section{Introduction}

1 Le milieu marin et sa biodiversité font l'objet de dispositifs de protection et de conservation tels que la création d'Aires Marines Protégées (AMP) définies en France dans la loi du 14 avril 2006. Une AMP est « un espace délimité qui répond à un objectif de protection de la nature à long terme, non exclusif d'un développement économique maîtrisé, pour lequel des mesures de gestion sont définies et mises en œuvre. » (Agence des AMP 2015). L'AMP du Prêcheur en Martinique a été créée par délibération du Conseil Régional de Martinique le 14 octobre 2014. Les pêcheurs, installés au Prêcheur depuis des générations, pratiquent une pêche artisanale, à bord de petits bateaux motorisés, au large et une pêche côtière. Même si, à ce jour, aucune structure de gestion n'existe pour la faire fonctionner, les effets probables de l'AMP du Prêcheur sur les pêcheurs sont à la fois d'ordre économique du fait de l'altération de leurs revenus (Failler et al. 2013:15) et symbolique par la remise en cause de leurs pratiques traditionnelles et de leur place d'usagers professionnels de la mer.

2 L'objectif de l'article est de présenter les résultats de l'enquête sociologique menée auprès des pêcheurs du Prêcheur ${ }^{2}$. Celle-ci visait à [1] étudier la connaissance des pêcheurs relative à la biodiversité marine et côtière et à la capacité de résilience des écosystèmes des AMP vis-à-vis des changements globaux et [2] proposer des mécanismes de valorisation des savoirs traditionnels des pêcheurs. Des entretiens semi-directifs portant sur l'activité de pêche (ses conditions, ses contraintes) et un relevé des observations par les pêcheurs de l'évolution de l'écosystème marin ont été effectués dans la commune du 
Prêcheur en 2016. L'analyse transversale des données apporte des éléments de connaissance nouveaux à la littérature dans la mesure où, depuis les travaux de Price (1964), peu d'études sont consacrées à la pêche traditionnelle caribéenne et aux savoirs détenus par les pêcheurs. Cet article permet de questionner, de manière inédite, le rapport des populations aux écosystèmes marins.

Dans une première partie, le cadre théorique ancre l'analyse dans une perspective anthropologique et économique. Dans une deuxième partie, sont précisées la méthode de recueil de données et les caractéristiques sociologiques des enquêtés. Dans une troisième partie, sont présentées les connaissances multidimensionnelles détenues par les pêcheurs sur la biodiversité marine, les capacités mobilisées par les pêcheurs pour évaluer l'évolution du milieu marin et les fonctions à travers lesquelles elles seraient potentiellement valorisables. Dans la quatrième partie, l'analyse est mise en perspective avec la littérature pour identifier les enjeux de la reconnaissance des savoirs traditionnels locaux.

\section{Cadre théorique}

4 La thématique des savoirs locaux et des savoirs traditionnels a été propulsée sur la scène internationale par la déclaration de Rio en 1992 qui apporte une reconnaissance des savoirs des populations locales dans la gestion de l'environnement. En 1993, la Convention sur la diversité biologique considère les savoirs traditionnels comme un patrimoine commun de l'humanité et propose un partage équitable des avantages qui découlent de la conservation et de l'utilisation durable de la diversité biologique (Carneiro da Cunha 2012).

5 Des anthropologues et des ethnologues ${ }^{3}$ de la nature ont alors parcouru la planète, dans le sillage des écologues et biologistes. Leurs travaux ont permis de poursuivre l'analyse des liens complexes entre nature et culture et de mettre en évidence l'existence des savoirs traditionnels au sein des populations vivant dans des zones particulièrement vulnérables du point de vue des menaces environnementales. En reconnaissant d'une part que les pratiques révèlent un processus d'adaptation, transmis entre générations, des relations entre les humains et leur environnement (Berkes et al. 2000 : 1252) et d'autre part que « la légitimité du savoir est liée à son inscription dans une lignée, indissociable de la relation à un territoire » (Roué \& Nakashima $2002: 380$ ), les anthropologues ont jeté les bases de la nécessaire implication des populations dans la production des savoirs écosystémiques. Même si le systématisme entre savoir traditionnel et conservation n'existe pas (Dove 2006), cette posture introduit le caractère indispensable de démarches participatives dans le domaine de la protection de la biodiversité contenant, parfois, pour les populations locales, une reconfiguration de leurs pratiques.

6 Au début des années 2000, l'urgence de la protection et de la conservation de la biodiversité en lien avec les changements climatiques a concentré toutes les énergies sans pour autant percevoir les revendications identitaires face à l'uniformité de la globalisation (Roué 2012). L'ethnoécologie et l'anthropologie maritime s'accordent alors pour définir les savoirs locaux comme des savoirs naturalistes, en lien avec la faune et la flore (Geistdoerfer 2007), «fondés sur des connaissances concrètes, hétérogènes et non formalisées " (Alphandéry \& Fortier 2005: 166), issus à la fois de l'observation quotidienne de la nature et de ses changements et du «feedback learning " (Berkes et al. 2000 : 1251). Les savoirs traditionnels comportent un élément supplémentaire dans la 
mesure où ils sont perçus comme un héritage, porteurs de modes d'acquisition et de productions spécifiques. En étudiant "la coexistence entre les sociétés et la nature", l'anthropologie de la nature cherche à comprendre le lien entre biodiversité et culture à travers l'analyse des pratiques et savoirs naturalistes des populations, leur transmission et l'impact sur la biodiversité et sa gestion (Dumez et al. 2014 : 3). Il s'agit alors d'identifier les « logiques de classifications des savoirs ", les principes et les critères qui régissent ces catégorisations, « les systèmes de repérages des données » (Bromberger 2006) et l'usage qui en est fait dans la mesure où la connaissance induit des manières d'agir, ici les pratiques de pêches. La plupart du temps, ces études sont menées de concert avec des recherches "classiques " visant à produire un diagnostic environnemental permettant d'établir l'état de dégradation de la biodiversité en vue d'une gestion durable des ressources.

7 Les processus de concertation et de gestion intégrée (Guineberteau et al. 2006) sont complexes à mettre en œuvre dans le domaine de la protection de l'environnement (non exclusivement). À travers la planète, depuis une vingtaine d'années, les études attestent de réussites ou d'échecs qui renvoient à quatre dimensions essentielles. La tension entre l'intérêt local et un intérêt global peut engendrer des incompréhensions entre les populations locales et les promoteurs de la conservation. Les savoirs "profanes", « locaux » se trouvent en concurrence avec les savoirs dits « scientifiques». Les différents niveaux d'échelons décisionnels tentent d'être présents dans les processus de gouvernance. Les activités traditionnelles peuvent être considérées comme une des causes de la dégradation de la biodiversité.

8 C'est dans ce contexte, que sont pensés les paiements pour services éco-systémiques (PSE), une notion économique qui propose d'abord d'identifier les connaissances détenues par les acteurs locaux sur la biodiversité et ses capacités de résilience, puis de concevoir un système de rétribution des services rendus par l'homme à la biodiversité. Dans la littérature, il existe une utilisation indifférenciée de la notion de service éco-systémique et de service environnemental qui peut s'expliquer par la proximité sémantique des deux notions mais aussi par le fait que la finalité des services environnementaux est bien la conservation de l'écosystème. Pourtant, les services éco-systémiques (SES) se distinguent des services environnementaux (SEV) par le statut du fournisseur. Les SES sont fournis par le milieu marin à la population (MEA 2005) alors que les SEV émanent de l'action des populations sur la protection et la conservation des écosystèmes (Bonin \& Antona 2012). Le bénéficiaire des SEV a un double statut: la biodiversité elle-même et les utilisateurs des SEV. Dans une logique économique de fourniture de service, l'identification des SEV ouvre ainsi la voie à une rétribution. Un PSE se décrit comme une transaction volontaire entre un fournisseur identifié et un acheteur pour un service défini et effectif (Wunder 2005, cité par Bonin \& Antona $2012: 6$ ).

9 En conjuguant la perspective anthropologique des savoirs locaux en lien avec la protection et la conservation de la biodiversité et l'approche économique des marchés de services, l'étude menée au Prêcheur apporte des éléments de réflexion sur la capacité d'un groupe professionnel à valoriser ses savoirs sur le milieu marin mais aussi sur la dimension interdisciplinaire indispensable aux démarches de gestion des ressources naturelles. Les témoignages recueillis auprès des pêcheurs permettent d'illustrer ce cadre théorique et de le prolonger dans une optique de cogestion de la biodiversité visant à déterminer les modalités d'une rétribution des SEV rendus par les pêcheurs. 


\section{Méthode et présentation des enquêtés}

10 L'enquête conduite en 2016 auprès de 16 marins-pêcheurs a été construite dans une perspective compréhensive et qualitative. L'échantillon représente environ $50 \%$ de l'effectif des pêcheurs déclarés en activité. Le recrutement s'est effectué selon la méthode de l'échantillon en cascade qui vise à questionner des personnes aux caractéristiques hétérogènes appartenant à un groupe « homogène » (Pires 1997). Notre installation sur le site du Prêcheur a permis les prises de contact individuelles.

11 Les entretiens semi-directifs menés en face à face se sont déroulés sur les lieux de travail, à proximité des bateaux, pendant les heures creuses de pêche. La grille d'entretien contenait plusieurs items : les caractéristiques socio-démographiques (âge, genre, lieu de résidence, nationalité, langue), le statut et le parcours professionnel (date et modalités d'installation, expériences de travail), l'activité de pêche (durées, espèces, lieux, techniques), les observations de l'état et de l'évolution des espèces et du milieu marin, la perception des effets des changements climatiques sur la ressource, les solutions et stratégies envisagées face aux défis rencontrés. Lors des entretiens, les pêcheurs ont été sollicités pour cartographier leurs observations, un exercice proche «des cartes mentales » utilisées par Teh et al. (2012) avec les pêcheurs de Malaisie. S'inspirant de la démarche de Sundaram et al. (2012) pour évaluer les impacts d'espèces invasives sur la forêt en Inde, « les réponses ont été juxtaposées aux données écologiques » relevées par les scientifiques. L'analyse transversale et thématique des données permet d'accéder aux connaissances mobilisables par les pêcheurs au profit de la conservation de l'espace marin.

12 L'échantillon de 16 pêcheurs se caractérise par une diversité d'appartenance (âge, type de pêche, durée d'activité), assurant un large spectre de réponses et un accès aux différentes catégories existantes au sein du groupe professionnel. Il faut souligner toutefois qu'une partie des pêcheurs farouchement opposés à la création de l'AMP n'a pas souhaité participer à l'enquête.

13 Les 16 personnes rencontrées sont âgées de 30 à plus de 65 ans. Ce sont tous des hommes (aucune femme n'exerce le métier au Prêcheur). Quatorze sont en activité, un en invalidité et un retraité. Quinze sont issus d'une famille de marin-pêcheur. La moitié d'entre eux est installée depuis 20-30 ans. La moitié cumule la pêche au large et la pêche côtière avec les casiers. L'autre moitié conjugue pêche au large et la pêche au filet et/ou à la senne (pêche côtière traditionnelle). Quel que soit le type de pêche, les pêcheurs utilisent des petites embarcations motorisées (principalement des yoles en bois et quelques yoles en fibre de verre) et partent le plus souvent seuls en mer (Figures 1, 2 et 3). Sur les 14 pêcheurs en activité, seulement trois cumulent une autre activité (excursions touristiques). Tous passent la plupart de leur temps en mer ou préparent leur départ. Ainsi, leurs témoignages reposent sur une pratique régulière et ancienne. 
Figure 1 : Pêche à la senne en Martinique

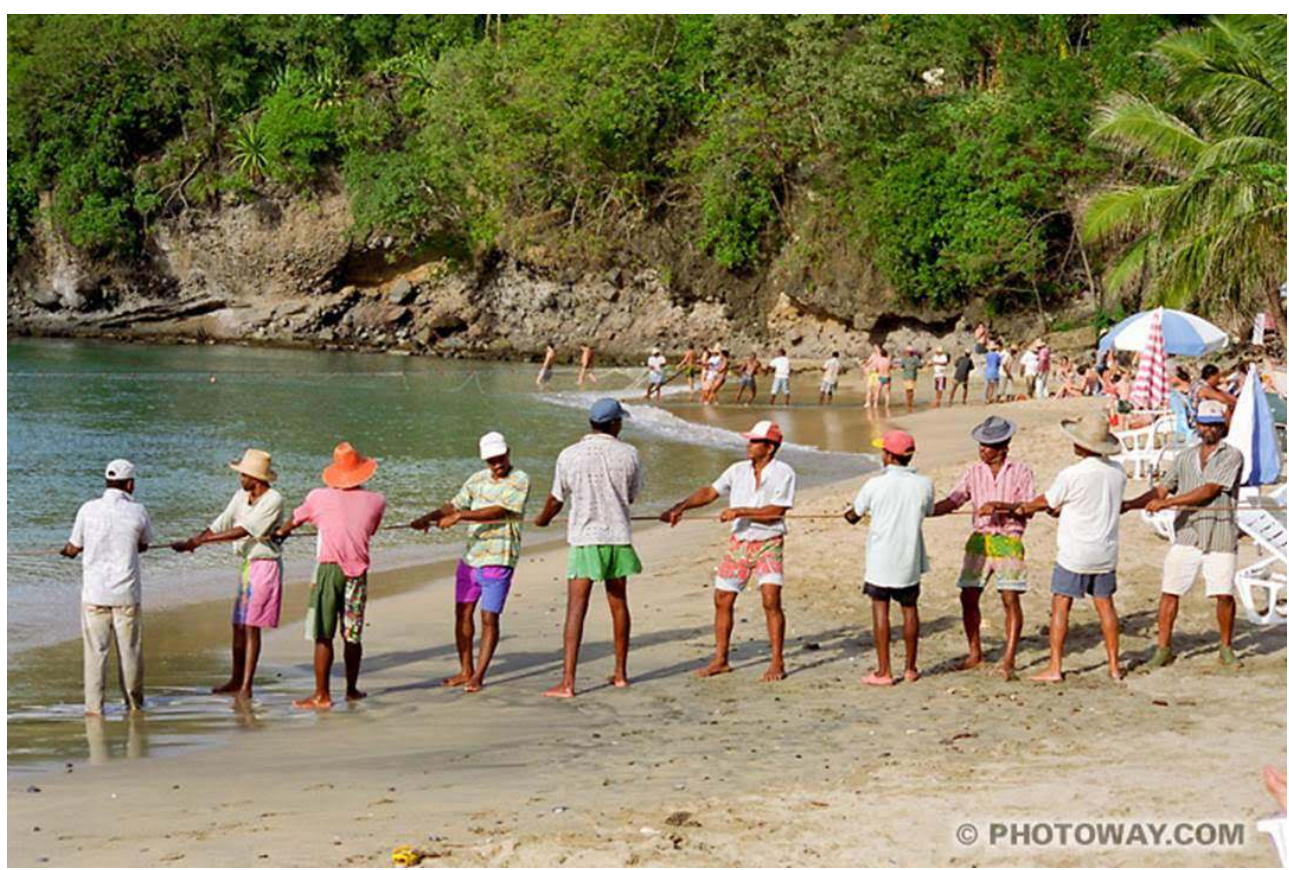

Figure 2 : Bateau traditionnel transportant un casier

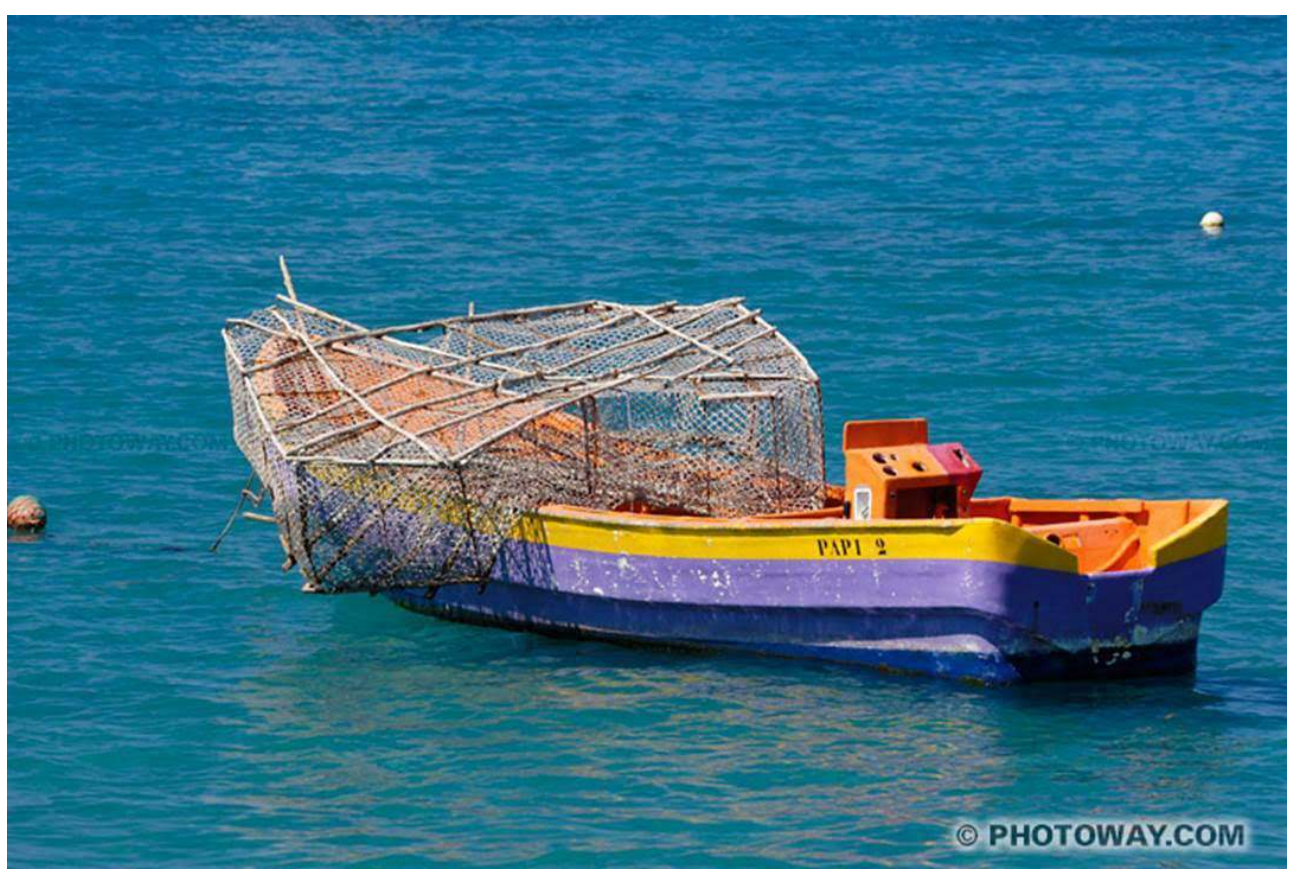

(c) M. Thirot 


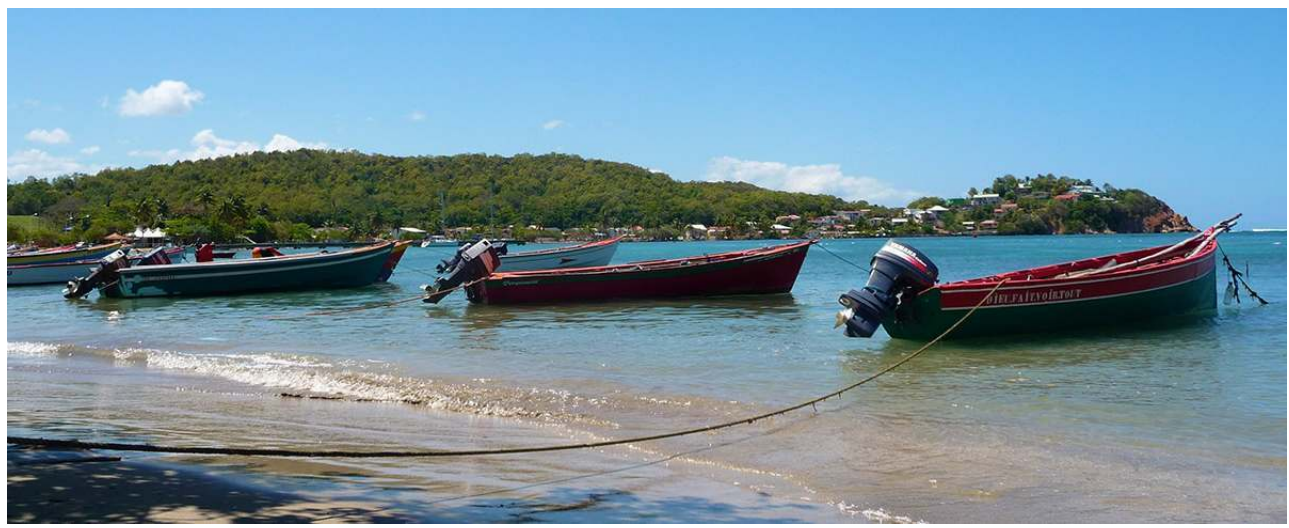

(c) E. Trégarot

\section{La connaissance du milieu marin à travers l'activité de pêche}

Les savoirs détenus par les pêcheurs aboutissent aux mêmes constats que ceux répertoriés dans l'étude préalable à la création de l'AMP conduite en 2011, concernant les techniques de pêche et les espèces capturées (IMPACT-MER et al. 2011). Les indicateurs utilisés pour construire le diagnostic halieutique et dresser l'état de dégradation de l'environnement sont identiques (Gay Wiber et al. 2012). On note une distinction majeure en termes de vocabulaire, les pêcheurs utilisant essentiellement le langage commun alors que le diagnostic environnemental cite exclusivement les noms latins des espèces. Les territoires de pêche déclarés par les pêcheurs semblent plus vastes que ceux répertoriés par les experts scientifiques (voir les cartes officielles en annexe 1). Les périodes de recueil de données diffèrent sensiblement. 


\section{Carte 1 : Zones de pêche sur le littoral de la commune du Prêcheur}

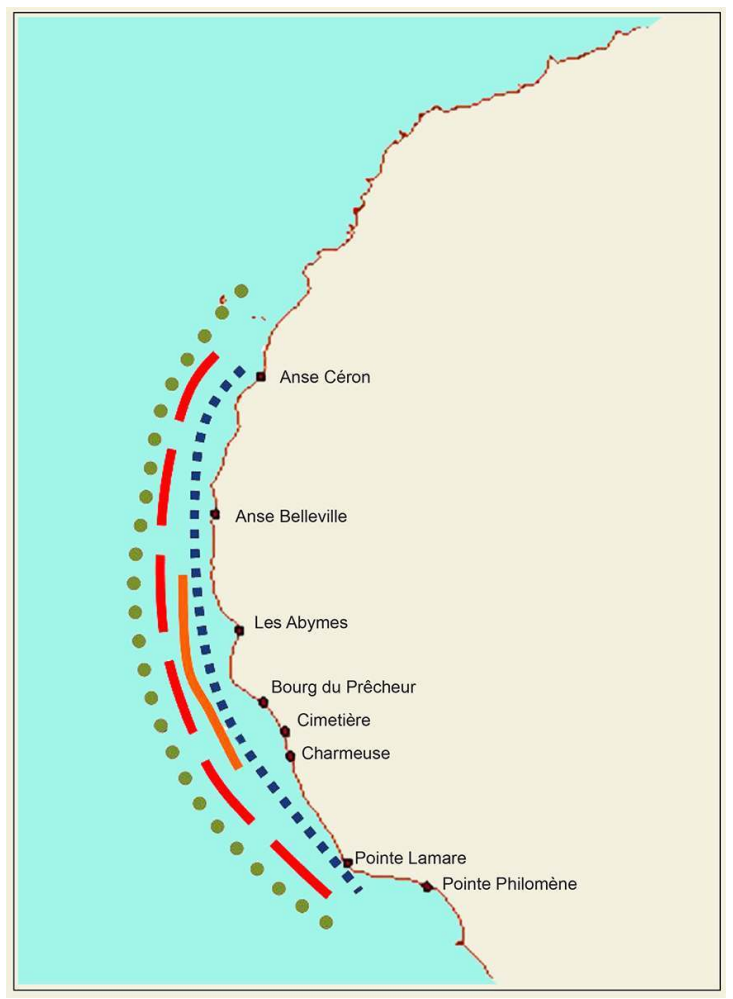

La senne de plage (carrés en pointillés bleus) et les filets de surface à BALAous (traits long rouges) sont déployés de l'anse Céron à la pointe Lamare. Les filets de fonds, trémails et autres (trait orange continu), sont disposés en face des Abymes et le bourg du Prêcheur. Les nasses sont déployées depuis le rocher de la Perle jusqu'à la pointe Lamare. Les DCP sont mouillés en dehors de la zone d'étude pour la réserve

Source : Régnier-Bohler (in Failler et al, 2011, p.18). 
Carte 2 : Les zones de la réserve marine du Prêcheur

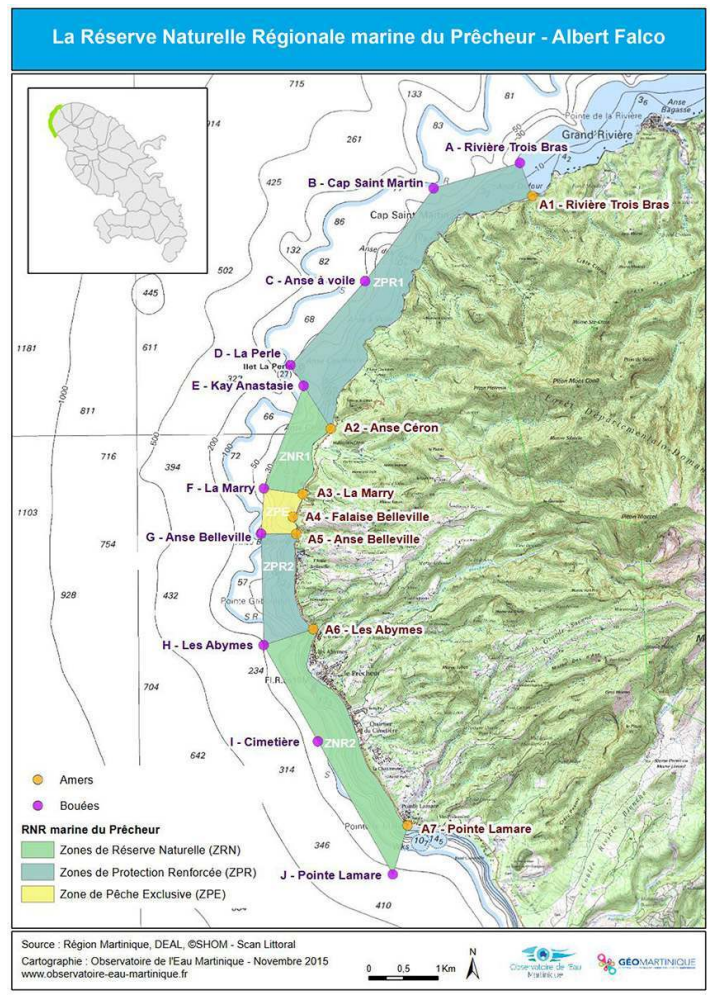

En effet, les connaissances des pêcheurs sur le milieu marin sont issues d'une présence quotidienne en mer (les sorties ont lieu toute la semaine et sur une amplitude horaire variant de 8 à 12 heures) et de la transmission des données par les pairs au cours de l'apprentissage du métier (tous les pêcheurs en activité sont issus d'une famille de pêcheurs). À ce titre, ces savoirs naturalistes portent sur l'éthologie des espèces, les facteurs abiotiques, hydrauliques, océanographiques et climatiques qui «régissent la présence et l'abondance du poisson sur les lieux de capture » (David 2008).

Les connaissances des pêcheurs portent d'abord sur les caractéristiques physiques (taille, couleur) et les habitudes des poissons. Des classifications des espèces (voir les noms scientifiques en annexe 2) sont opérées selon leurs lieux de reproduction et de présence par rapport au littoral (" poissons rouges » à la côte ${ }^{4}$, " poissons pélagiques » ${ }^{5}$ au large) et en fonction de la profondeur où ils vivent (« balaous » en surface, " poissons de fond » ${ }^{6}$ à la côte). La confection des différents types d'appâts (végétaux, poisson) et les lieux de présence des juvéniles permettent aux pêcheurs d'identifier la chaîne alimentaire selon les espèces (herbiers ou alevins). Les grands prédateurs sont spécifiés (requins, poissonlions). En fonction des périodes d'abondance de la pêche (septembre à avril) ou de " disette » (mai à septembre), les pêcheurs identifient les périodes de reproduction et les déplacements de chaque espèce de poissons. Ainsi, les lieux de pêches sont de plus en plus variés, lointains; le périmètre de pêche (donc d'observation) s'étend en taille et en distance depuis une vingtaine d'années.

L'observation des localisations et des déplacements des espèces se conjugue à celle d'éléments océanographiques. La direction et l'intensité des courants ont des effets sur la présence des espèces de surface (comme « les poissons volants » et les « balaous ») ou à la côte (« coulirous », « maquereaux », " poissons rouges »). Des connaissances climatiques 
telles que la température de l'eau, la force du vent, l'amplitude de la houle sont également mobilisées pour comprendre les déplacements des espèces mais aussi les impacts de ces facteurs sur la reproduction (éclosion des œufs, qualité des habitats). Enfin, les lunaisons sont prises en compte pour intensifier ou non la pêche à la côte.

Ces savoirs "empiriques", issus d'observations pratiques, induisent les comportements et les décisions des pêcheurs, soumis à des ressources instables. Toutefois, en identifiant les relations de cause à effet entre la présence et le type de ressources et les conditions de son milieu de vie, ces pêcheurs détiennent «une vison intégrée et systémique du fonctionnement des écosystèmes " (Barthélémy 2005 : 5). L'ancrage quotidien, local et historique des pêcheurs leur permet une accumulation de connaissances, sources d'interprétation des processus écologiques à l'œuvre dans le milieu marin. Compte tenu de la diversité des espèces présentes, de la discontinuité et de l'instabilité des caractéristiques du milieu marin, les données recueillies changent de manière quotidienne mais aussi saisonnière et annuelle. Les pêcheurs doivent ainsi sans cesse s'interroger, poser des hypothèses et tenter d'aller les vérifier in situ. L'exercice est d'autant plus important pour apprécier l'évolution du milieu marin et ses capacités d'adaptation et de résilience face aux changements climatiques.

19 Partant du constat d'une baisse des quantités de poissons pêchées, de la nécessité de pêcher plus longtemps et plus loin et de cumuler pêche à la côte et pêche au large pour garantir un certain niveau de rentabilité, les pêcheurs détiennent aussi des connaissances sur les espèces en danger, l'état du milieu marin et l'aspect physique de la côte.

Plusieurs paramètres sont mobilisés par les pêcheurs pour évaluer l'état de la ressource. La diminution de la taille des poissons (dorades, marlins), la moindre ampleur des bancs (de thon) au large et à la côte (poissons rouges), la rareté des balaous et la quasidisparition des poissons volants constituent des indicateurs de comparaison des équilibres sur une échelle temporelle longue (certains exercent depuis 30 ans). Ces évolutions sont sources d'inquiétude sur la viabilité de l'activité à court et moyen terme mais constituent aussi des occasions pour chercher les causes de ces changements.

21 Les évolutions observées dans la distribution et la santé des prises ou l'absence de capture sont situées dans un espace-temps précis et consensuel et associées à deux types de facteurs. La baisse du rendement de l'activité et la rareté de certaines espèces semblent être progressives depuis la fin des années 2000 (aucune donnée officielle ne permet de corroborer cette affirmation dans la mesure où il n'existe pas de relevés périodiques).

Le premier type de facteurs explicatifs concerne les changements du milieu marin. D'abord, les pêcheurs détiennent une connaissance des interrelations entre les espèces. L'apparition accidentelle d'un prédateur redoutable (poisson-lion) dans les eaux de la Caraïbe constituerait la principale raison de la diminution des espèces pêchées de manière traditionnelle (filets à la traîne, casiers, sennes). Ensuite, les pêcheurs ont acquis une connaissance des interactions entre la ressource halieutique et les espèces végétales. Ainsi, les invasions d'algues sargasses seraient la cause de l'évolution des tailles et de la disparition de certaines espèces (dorades, poissons volants). Puis, la connaissance des fonds marins (morphologie, coraux) et de leur configuration physique à proximité de la côte permet d'établir un lien entre la baisse de la pêche côtière et l'érosion.

Ces éléments explicatifs apportés par les savoirs des pêcheurs comprennent ainsi des facteurs locaux mais aussi globaux. Ils observent en effet l'augmentation du niveau et de la température de la mer et leurs corollaires c'est-à-dire une diminution du plancton et le 
blanchissement des coraux. Ils observent aussi « un dérèglement climatique » c'est-à-dire une répartition des cycles de saison différente d'une année à l'autre avec des incidences sur la reproduction, la présence et l'abondance des poissons. En comparant d'une part leur calendrier et leur lieu de pêche et d'autre part les variations océanographiques, climatiques et halieutiques, ils élaborent à court et à moyen terme des hypothèses de causalité voire de prédiction entre phénomènes globaux et réalités locales.

Le second type de facteurs explicatifs de l'évolution constatée concerne les activités humaines. Certains reconnaissent que le changement et l'amélioration des techniques (augmentation de la taille et de la puissance des moteurs), l'intensification de l'activité de pêche (dispositifs de concentration de poissons ${ }^{7}$ DCP, bateaux plus performants), la concurrence géographique (entre pêcheurs) ont des incidences sur la pêche, renforçant la nécessité d'une intervention rapide et l'introduction d'une pêche durable et responsable. Selon le diagnostic établi par Failler et al (2011), en 2009, les captures s'élèvent à 85 tonnes de poissons pour la pêche au large et à 22 tonnes pour la pêche côtière (concernée par l'AMP).

Face à ces changements, les propositions sont diverses et portent sur l'activité de pêche en elle-même. L'adaptation des pratiques de pêche à travers la diversification, la polyvalence des techniques, la flexibilité en fonction de l'abondance et l'augmentation des dispositifs de concentration de poissons (DCP) permettraient, selon les pêcheurs, d'assurer des revenus de substitution à la pêche traditionnelle la plus nocive pour la ressource à la côte. Ces évolutions supposent d'une part la mise en place et le respect de zones d'exclusion et d'autre part un accroissement de la taille des bateaux donc des investissements lourds. Ces mesures visent un maintien de leur revenu tout en assurant la continuité de la biodiversité et du stock et la protection des habitats.

Sur les impacts des changements climatiques globaux, les pêcheurs ne peuvent qu'en constater les conséquences et les inclure dans leurs pratiques pour tenter de les atténuer. Ils mettent en place des stratégies d'adaptation soit, pour la plupart, en multipliant les sorties au large, soit, pour certains, en proposant des excursions touristiques visant à valoriser le patrimoine maritime, culturel et historique local.

\section{La valorisation des connaissances des pêcheurs}

Le milieu marin constitue un patrimoine maritime mobilisant des connaissances spécifiques, applicables à une zone géographique spatialement circonscrite mais vaste. Cet écosystème complexe se caractérise par une biodiversité soumise à des contraintes naturelles, climatiques et humaines. Considérés comme une ressource vulnérable, les services éco-systémiques rendus à l'homme, à travers la pêche notamment, sont menacés, tout comme leur fonction d'approvisionnement. Face à ce défi, et pour conserver et protéger le milieu marin, l'encadrement et la gestion des activités de pêche apparaissent comme des solutions inévitables.

Depuis les trente dernières années, l'activité a déjà connu de nombreuses évolutions réglementaires. L'interdiction des captures de tortues a été adoptée en 1993. La taille des mailles des filets et des casiers a été augmentée pour éviter de capturer les juvéniles. Les DCP visent à déplacer le territoire de pêche vers le large et diminuer la pression sur le plateau insulaire. 
29 Malgré ces différentes solutions, l'écosystème marin du Prêcheur est toujours en danger. La pêche artisanale pratiquée fait perdurer les traditions ancestrales tout en ayant associé de nouvelles techniques ainsi la pression sur la ressource ne faiblit pas. Parallèlement la pêche procure toujours des moyens élémentaires de subsistance aux pêcheurs et à leurs familles. Les activités de pesca-tourisme et de plongée ont fait leur apparition. L'impact de ces activités humaines nécessite de nouveaux efforts, notamment de la part des pêcheurs, qui risquent d'affecter leurs revenus déjà modestes, compte tenu du caractère aléatoire et instable de leurs prises.

L'état des lieux de leurs connaissances vise ainsi leur reconnaissance et leur valorisation, voire une gratification, sous la forme de " paiements de services éco-environnementaux " de quatre types de fonctions : la position occupée par les pêcheurs, la permanence de leur présence, la superficie parcourue ainsi que la superficie connue et surveillée constituent des conditions idéales pour collecter des données essentielles sur l'état du milieu marin et son évolution, sur les ressources halieutiques et leur place dans la chaine trophique (incluant la côte).

31 Pour être validées, les données doivent nécessairement être contextualisées et comparées. Les différents lieux de pêche constituent des postes d'observation. Les captures mais aussi leur absence révèlent pour chaque zone fréquentée la présence des espèces, de leur nourriture, de leur habitat, de leur mode de déplacement. Les techniques utilisées nécessitent de "sonder " et d'explorer la mer à des profondeurs variées et donnent accès à l'observation des fonds marins, leur richesse et leur évolution et imposent la prise en compte de données de courantologie, de climatologie et d'océanographie. L'observation permet de comprendre les caractéristiques de chaque territoire.

32 Chaque écosystème évolue dans le temps. Ainsi les observations sont renouvelées et actualisées en fonction des saisons et des lunaisons, faisant appel à des données météorologiques. Les données recueillies tiennent compte de temporalités courtes (à la journée, à la semaine) et longues (plusieurs années). Les pêcheurs sont donc d'abord de fins observateurs d'un écosystème évolutif.

33 La gestion durable du milieu ne peut incomber aux seuls pêcheurs dans la mesure où ils ne sont pas les seuls utilisateurs, dans la mesure aussi où ils ne sont pas les seuls dépositaires de la préservation de ce patrimoine. Toutefois, le rapport qu'ils entretiennent avec « la mer nourricière » les place dans une position privilégiée d'analyste . L'analyse de ces observations comporte des dimensions spatiales et temporelles essentielles à la compréhension de l'évolution de l'écosystème. Pour cela, les pêcheurs se livrent à une méthode proche de l'analyse à composantes multiples utilisée en statistiques pour distinguer les variables dépendantes et indépendantes d'un phénomène. La prise en compte de l'interaction et de l'interdépendance de ces données nécessite une lecture dynamique et une approche pluridisciplinaire.

34 La gestion d'une AMP porte sur l'encadrement de l'activité, la définition de zones de restriction ou d'interdiction de pêche, leur durée et le suivi de l'évolution des ressources. Les pêcheurs peuvent donc être parties prenantes des évaluations quantitatives et qualitatives des mesures mises en place, tout au long du processus de décision qui comporte des phases de diagnostics, de suivis, d'interprétations des résultats et de réajustements. 

relatives à la biodiversité, son évolution, ses capacités de résilience face aux changements climatiques et ainsi réfléchir à des modalités de «paiement pour services environnementaux (PSEV)». géographiquement et comportent une dimension historique dans la mesure où ils sont acquis par transmission. Au Prêcheur, les pêcheurs sont nés et ont grandi dans une famille de pêcheurs (filiation) mais aussi dans la « communauté de la pêche » (proximité), installée dans cette commune depuis des centaines d'années. Les connaissances sont acquises par l'observation et la reproduction de gestes, par l'écoute des récits de pêche et par la présence répétée des garçons dans les bateaux. Cette immersion continue et constante crée une «intimité avec le milieu marin » et permet de le découvrir. "Leurs savoirs sont basés sur l'expérimentation » canalisée et guidée par les aînés (Roué $2012: 8$ ). Selon Dubost (1996 : 26) :

« les techniques de pêche révèlent l'interaction des pêcheurs avec le milieu marin et supposent un savoir-faire technique, un savoir naturaliste, la connaissance des fonds marins et des territoires de pêche. [....] Les outils utilisés représentent une trace de la transmission et une adaptation des matériaux ». 

ressources diffèrent entre les pêcheurs et les experts environnementaux, les résultats convergent en termes de diagnostic. La dissension et les inquiétudes résident dans les solutions à mettre en œuvre dans la mesure où les postures laissent apparaître des intérêts divergents. L'enjeu est alors de poser un autre regard sur l'activité des pêcheurs, notamment ceux qui sont directement impactés par les zones de protection même si toute la profession est concernée.

41 En effet, les savoirs naturalistes mis en évidence dans la section précédente peuvent être valorisés à travers la notion de services environnementaux (SEV). Froger et al. (2012) en proposent une typologie. La notion de «service-externalité » est la plus répandue pour évoquer l'impact non intentionnel mais réel de l'activité de l'homme sur les SES. L'embellissement, la protection, l'entretien et l'aménagement du milieu naturel sont considérés comme des externalités positives. La seconde approche est celle du «service activité » mis en place et géré par les services publics (dans le domaine de l'eau par exemple). La troisième approche qualifie le «service-produit joint » pour désigner les nouvelles filières de commerce équitable ou « bio ».

Leur activité étant directement liée au milieu marin, les pêcheurs peuvent être considérés comme des fournisseurs de service-externalité. Le défi étant de changer d'image dans la mesure où leur activité est globalement perçue comme négative vis-à-vis des ressources, argument fondateur dans la mise en place de l'AMP. Les résultats de l'enquête ont démontré les connaissances des pêcheurs vis-à-vis du milieu marin et des ressources halieutiques, des interactions entre les conditions climatiques et l'état de la mer et des fonds marins. Toutefois, à l'heure actuelle, l'utilisation de ces connaissances vise essentiellement à gérer et organiser leur propre activité. La valorisation de ces connaissances vise leurs intérêts professionnels. À terme, si les conditions de production et de commercialisation liées au commerce équitable étaient réunies, on pourrait imaginer que les produits vendus par les pêcheurs puissent obtenir un label, nécessairement adapté à la pêche artisanale caribéenne ${ }^{8}$. la connaissance des savoirs locaux des pêcheurs, assurer et promouvoir la conservation du milieu. L'approche économique institutionnaliste des PSEV proposée par Wunder (2005) les envisage comme une compensation de services rendus pour « inciter à articuler les décisions individuelles et collectives avec l'intérêt social de la gestion durable des ressources naturelles et la conservation de la biodiversité » (Froger et al. 2012 : 8). Conçus dans une logique de marché, les échanges volontaires entre fournisseurs et bénéficiaires des services peuvent ainsi faire l'objet de négociations, obligeant toutefois les fournisseurs à s'engager dans des activités d'usage spécifiques et à tenir compte de la demande pour fixer leur prix. Par ailleurs, la compensation s'effectue dans « une logique d'additionnalité », le service devant apporter un gain à la biodiversité et dans "une logique de conditionnalité » assurant l'effectivité du service, sa fourniture devenant obligatoire (Froger et al. 2012 : 8). L'activité devant être à la fois produite et utile à la biodiversité, le problème de l'évaluation de cette efficacité reste entier.

Muradian et al. (2010) contestent la logique purement rationnelle de cette définition pour trois raisons essentielles liées à la problématique de la conservation de la biodiversité et de son caractère complexe, diffus et temporel. En premier lieu, les SEV sont difficilement quantifiables. Comment évaluer l'impact de la participation des pêcheurs aux instances 
délibératives de gestion de l'AMP sur la protection de l'écosystème marin? À quelle échéance, les actions d'éducation menées en direction des jeunes générations porterontelles leur fruit? L'efficience à court terme des services fournis ne peut porter que sur leur effectivité et sur un rapport coût/avantage sur la base d'un scénario minimaliste (Pascual et al. 2010). En second lieu, les fournisseurs ne sont pas tous égaux. Les activités touristiques ont le vent en poupe alors que la situation économique des pêcheurs est vulnérabilisée par la baisse des ressources, le manque d'organisation commerciale de la filière, des charges sociales considérées comme excessives. Le processus de PSEV suppose une réflexion en terme d'équité sur les montants et les destinateurs de la compensation. Enfin, l'incitation ne doit pas être toujours financière. Muradian et al. (2010) alertent sur la prise en compte de ces contraintes sociales et des perceptions de la relation entre les usagers et la nature dans la conception des PSEV. Au Prêcheur, à l'heure actuelle, la plupart des pêcheurs ne souhaitent pas changer d'activité professionnelle du fait de l'incertitude que de telles reconversions représentent. Si la compensation peut être monétaire, elle doit être suffisamment convaincante pour renoncer à des pratiques ancestrales. Comment diminuer l'incertitude liée à la fourniture du service? Faut-il imaginer le paiement sous forme de forfaits? Comment prendre en compte leur attachement à une pêche artisanale?

\section{Conclusion}

45 L'intensité du rapport des pêcheurs "artisanaux » à la mer leur donne une place spécifique dans le lien entre activités humaines et écosystèmes. Alors qu'ils sont la plupart du temps considérés comme «nuisibles» et, en partie, responsables de dégradations visibles du milieu marin, il importe de rappeler que les pêcheurs puisent leurs revenus de l'exploitation des ressources halieutiques, tout en s'exposant à des conditions de travail dangereuses. Dans une perspective de conservation de la biodiversité, l'enquête menée en Martinique permet de changer le regard sur ces acteurs économiques et de les considérer comme des experts naturalistes. Les marins-pêcheurs du Prêcheur en Martinique sont bien détenteurs de savoirs naturalistes locaux attestant de compétences cognitives, techniques et scientifiques.

La mise en cuvre d'une gestion durable des ressources marines suppose le développement de systèmes concrets de gratification assurant la reconnaissance et la valorisation de ces savoirs et des acteurs qui les détiennent. Ces mécanismes de compensation doivent pouvoir prendre en compte deux types de services environnementaux rendus par les pêcheurs. Le premier repose sur un engagement vis-àvis de la protection du milieu marin et suppose un changement de pratiques. Le bénéficiaire direct est la biodiversité. Le second service consiste à exploiter les savoirs détenus en s'impliquant dans les opérations de gestion durable des ressources, à court et moyen termes. Le bénéficiaire est la société (plus globalement l'humanité) et plus précisément les destinataires des services rendus que sont les acteurs institutionnels, les autres usagers de la mer et les populations locales. Dans tous les cas, ces gratifications doivent répondre à des exigences de justice et de redistribution équitables.

D'un point de vue théorique, la notion d'expert naturaliste que nous avons tenté d'esquisser ouvre la voie à de nouvelles investigations empiriques et théoriques pour les différentes disciplines des sciences humaines et sociales et éclaire les démarches de gestion intégrée de la biodiversité. 
Cet article été produit avec le soutien financier de la Commission européenne dans le cadre du programme européen CARIPES « Paiements pour les services écosystémiques des aires marines protégées de la Caraibe " de l'Action préparatoire BEST (Régime volontaire pour la biodiversité et les services écosystémiques dans les territoires des régions ultrapériphériques et les pays et territoires d'outre-mer de l'Union européenne). Les opinions émises dans l'article ne reflètent pas nécessairement celles de la Commission européenne et n'anticipent en rien la politique de la commission dans ce domaine.

\section{BIBLIOGRAPHIE}

Agence des Aires Marines Protégées 2015 - Orientations : contextes, enjeux et pistes d'action. Groupe de travail pour la création d'un parc naturel marin en Martinique, Martinique, 8 p.

Alphandéry P. \& Fortier A. 2005 - Les savoirs locaux dans les dispositifs de gestion de la nature . In Bérard L. et al. (Ed.), Biodiversité et savoirs naturalistes locaux en France. CIRAD, IDDRI, IFB, INRA : 158-168.

Barthélémy C. 2005 - Les savoirs locaux : entre connaissances et reconnaissance. Vertigo - la revue électronique en sciences de l'environnement 6 (1), [En ligne] consulté le 18 octobre 2012. URL : http:// vertigo.revues.org/2997; DOI : 10.4000/vertigo.2997

Berkes F. Colding J. \& Folke C. 2000 - Rediscovery of Traditional Ecological Knowledge as Adaptive Management. Ecological Applications 10 (5) : 1251-1262.

Bonin M. \& Antona M. 2012 - Généalogie scientifique et mise en politique des services écosystémiques et services environnementaux. Vertigo - la revue électronique en sciences de l'environnement 12 (3), [En ligne] consulté le 13 juin 2016. URL : http://vertigo.revues.org/13147 ; DOI : $10.4000 /$ vertigo. 13147

Bromberger C. 2006 - Les savoirs des autres. Terrain [En ligne], 6, consulté le 13 juillet 2016. URL : http://terrain.revues.org/2890; DOI : 10.4000/terrain.2890

Carneiro da Cunha M. 2012 - Savoirs autochtones : quelle nature, quels apports ? Leçon inaugurale au Collège de France prononcée le 22 mars.

Conklin H.C. 1955 - The Relation of Hanunoo Culture to the Plant World. Ph. D. dissertation, Yale University. Ann Arbor, Michigan, University Microfilms Inc., 471 p.

David G. 2008 - La pêche côtière océanienne et son environnement. Le Journal de la Société des Océanistes 126-127, [En ligne], consulté le 10 juillet 2016. URL : http://jso.revues.org/4352

Dove M.R. 2006 - Indigenous People and Environmental Politics. Annual Review of Anthropology 35 : 191-208.

Dubost I. 1996 - De soi aux autres ... un parcours périlleux. La construction d'un territoire par les pêcheurs martiniquais. Thèse de doctorat d'anthropologie. $840 \mathrm{p}$.

Dumez R., Roué M. \& Bahuchet S. 2014 - Conservation de la nature : quel rôle pour les sciences sociales? Revue d'ethnoécologie 6, [En ligne] consulté le 01 avril 2016. URL : http:// ethnoecologie.revues.org/2089; DOI : 10.4000/ethnoecologie.2089. 
Failler P., Binet T. \& Borot de Battisti A. 2011 - Création de la réserve marine régionale du Prêcheur : Diagnostic socio-économique des écosystèmes marins et côtiers. Prêcheur, Rapport pour le Conseil Régional de la Martinique, Fort-de-France, Martinique, 92 p.

Failler P., Borot de Battisti A., Binet T. \& Violas L. 2013 - Conséquences de la création de l'aire marine régionale du Prêcheur (Martinique) sur la pêche martiniquaise. Études caribéennes 26, [En ligne] consulté le 17 juin 2016. URL : http://etudescaribeennes.revues.org/6644 ; DOI : 10.4000/ etudescaribeennes.6644

Freeman M. 1992 - The nature and utility of traditional ecological knowledge. Northern Perspectives 20 (1) : 7-12.

Froger G., Méral P., Le Coq J-F., Aznar O., Boisvert V., Caron A. \& Antona M. 2012 - Regards croisés de l'économie sur les services écosystémiques et environnementaux. Vertigo - la revue électronique en sciences de l'environnement 12 (3), [En ligne], consulté le 11 juillet 2016. URL : http:// vertigo.revues.org/12900; DOI : 10.4000/vertigo.12900

Gay Wiber M., Young S. \& Wilson L. 2012 - Impact of Aquaculture on Commercial Fisheries: Fishermen's Local Ecological Knowledge. Human Ecology 40 : 29-40.

Geistdoerfer A. 2007 - L'anthropologie maritime : un domaine en évolution: hors cadre traditionnel de l'anthropologie sociale. Zainak 29 : 23-38.

Guineberteau T., Meur-Ferec C. \& Trouillet B. 2006 - La gestion intégrée des zones côtières en France : mirage ou mutation stratégique fondamentale ?. Vertigo - la revue électronique en sciences de l'environnement 7 (3), [En ligne], consulté le 09 novembre 2012. URL : http:// vertigo.revues.org/2569; DOI : 10.4000/vertigo.2569

IMPACT-MER, OMMM, BIOS, CEMARE 2011 - Étude préalable à la création de la réserve marine du Prêcheur. Diagnostics écologique, socio-économique, usages et pressions. Analyse des enjeux. Rapport diagnostic pour le Conseil Régional Martinique, $217 \mathrm{p}$.

Johannes R.E. 1981 - Words of the Lagoon: Fishing and Marine Lore in the Palau District of Micronesia. Berkeley, University of California Press, $320 \mathrm{p}$.

Millenium Ecosystem Assessement 2005 - Rapport de synthèse version française. 59 p.

Muradian R., Corbera E., Pascual U., Kosoy N. \& May P.H. 2010 - Reconciling theory and practice: An alternative conceptual framework for understanding payments for environmental services. Ecological Economics 69 : 1202-1208.

Pascual U., Muradian R., Rodriguez L.C. \& Duraiappah A. 2010 - Exploring the links between equity and efficiency in payments for environmental services: a conceptual approach. Ecological Economics 69 : 1237-1244.

Pires A. 1997 - Échantillonnage et recherche qualitative : essai théorique et méthodologique. In Poupart, Deslauriers, Groulx, Laperrière, Mayer, Pires (Ed.) La recherche qualitative. Enjeux épistémologiques et méthodologiques. Montréal, Gaëtan Morin Éditeur : 113-169.

Price R. 1964 - Magie et pêche à la Martinique. L'Homme 4 (2) : 84-113.

Roué M. 2012 - Histoire et épistémologie des savoirs locaux et autochtones. Revue d'ethnoécologie 1, [En ligne], consulté le 23 mars 2016. URL : http://ethnoecologie.revues.org/813 ; DOI : 10.4000/ ethnoecologie. 813

Roué M. \& Nakashima D. 2002 - Des savoirs « traditionnels » pour évaluer les impacts environnementaux du développement moderne et occidental. Revue internationale des sciences sociales $3-173: 377-387$. 
Sundaram B., Krishnan S., Hiremath A.J. \& Joseph G. 2012 - Ecology and Impacts of the Invasive Species, Lantana cantara, in a Social-Ecological System in South India: Perspectives from Local Knowledge. Human Ecology 40 : 931-942.

Teh L.C.L., The L.S.L. \& Meitner M.J. 2012 - Preferred Resource Spaces and Fisher Flexibility: Implications for Spatial Management of Small-Scale Fisheries. Human Ecology 40 : 213-226.

Wunder S. 2005 - Payments for environmental services: some nuts and bolts. CIFOR Occasional paper, N $\circ 42$.

\section{ANNEXES}

Noms vernaculaires et scientifiques des poissons cités par les pêcheurs

\begin{tabular}{l|l}
\hline Balaou & Cololabis saira \\
\hline Coulirou & Trachurus trachurus \\
\hline Daurade & Coryphaena hippurus \\
\hline Maquereau & Equus lacertus \\
\hline Marlins & Istiophoridae \\
\hline Perroquets & Scaridae \\
\hline Poisson lion & Pterois volitans \\
\hline Poissons volants & Exocoetidae \\
\hline Requins & Selachimorpha spp. \\
\hline Thons & Thunnus spp. \\
\hline Vivaneau & Lutjanus campechanus
\end{tabular}

\section{NOTES}

1. Recherche financée par l'UE et le Conseil Régional de Martinique dans le cadre du projet BESTCARIPES (Régime volontaire pour la biodiversité et les services écosystémiques dans les régions ultrapériphériques et les pays et territoires d'outre-mer européens).

2. Nous adressons nos remerciements à toutes les personnes qui ont bien voulu nous accorder leur confiance pour mener à bien cette enquête.

3. On peut citer les pionniers : Conklin (1955) aux Philippines, Freeman (1992) Nord canadien et enfin Johannes (1981).

4. Principalement des vivaneaux.

5. Essentiellement des thons, des dorades, des marlins, des poissons volants.

6. Principalement des mérous, des perroquets.

7. Les dispositifs de concentration de poissons : ils consistent à ancrer ou faire dériver une ligne de flotteurs autour desquels vont s'agréger des algues ou des alevins et ainsi attirer les poissons pélagiques. Les pêcheurs concentrent alors leurs prises autour de ces dispositifs. 
8. Il existe un label français Promotion France pour la pêche en Atlantique et en Manche. Il vise à améliorer la traçabilité du poisson et les conditions de pêche durable (pratiques et saisonnalité). Au niveau international, le Marine Stewardship Council a créé son label mais il semble sujet à débat dans la mesure où il est associé à l'industrie agroalimentaire. http://www.greenpeace.org/ switzerland/Global/switzerland/fr/publications/

agriculture/2009_Agriculture_Brochure_LabelsAlimentaires.pdf

\section{RÉSUMÉS}

Cet article présente les résultats d'un travail de recherche conduit en 2016 auprès d'une communauté de pêcheurs du Nord-Ouest de la Martinique. Les entretiens semi-directifs menés en face à face par une sociologue ont permis de recueillir les éléments de connaissance des pêcheurs vis-à-vis de l'écosystème marin et des interrelations entre ses différentes composantes, ainsi que leurs savoirs relatifs à la résilience des écosystèmes des aires marines protégées vis-àvis des changements globaux.

En référence aux travaux d'anthropologie de la conservation et dans une perspective de gestion intégrée des ressources, l'analyse porte sur les savoirs locaux détenus par les pêcheurs sur la biodiversité marine et côtière. Sur la base des observations quotidiennes opérées, l'écosystème marin est analysé, par les pêcheurs, comme un système complexe soumis à une réalité multifactorielle nécessitant de leur part des compétences naturalistes et cognitives (éthologie des espèces, océanographie, climatologie, hydrologie). Les services environnementaux rendus par les pêcheurs traditionnels peuvent faire l'objet d'une reconnaissance par un système de paiements pour services écosystémiques.

This paper presents the results of a research project conducted in 2016 with fishermen based in the north west of Martinique, Caribbean. Semi-directing interviews led face-to-face by a sociologist provided elements of the fishermen empirical knowledge relating to marine ecosystems, the interdependence of their various components, as well as their knowledge on the resilience of marine protected areas ecosystems regarding global changes.

According to the anthropology of conservation and in a perspective of integrated resources management, the analysis focuses on local knowledge of fishermen of marine and coastal biodiversity. Based on daily observations, the marine ecosystem is analysed, by the fishermen, like a complex system submitted to a multifactorial reality, which demonstrates their naturalists and cognitive knowledge (species, oceanography, climatology and hydrology). The services provided by the fishermen can this be subject to a reward such as a payment for environmental services.

\section{INDEX}

Keywords : Martinique, local knowledge, fishermen, payments for environmental services Index géographique : Martinique

Mots-clés : savoirs locaux, pêcheurs, paiements pour services environnementaux 


\section{AUTEURS}

\section{MYRIAM THIROT}

Sociologue, Membre associé au LC2S (UMR CNRS 8053) Université des Antilles, Faculté de droit et d'économie de Martinique, BP. 7209, 97275 Schœlcher

thimyr@gmail.com

\section{PIERRE FAILLER}

Reader in Economics, Economics and Finance Group, Portsmouth Business School, University of Portsmouth, Richmond Building, Portland Street Portsmouth, PO1 3DE, UK United Kingdom pierre.failler@port.ac.uk

\section{JUSTIN DANIEL}

Professeur de science politique, directeur de l'UMR CNRS 8053, Université des Antilles, Faculté de droit et d'économie de Martinique, BP. 7209, 97275 Schœlcher d.justin@orange.fr 